\title{
Family and Social Support System in Reducing Neonatal Morbidity and Mortality among Neonates Born To Adolescent Mothers
}

\author{
Anne Wawire Kabimba*, Emmah Matheka, Sabina N. M. Wakasiaka
}

School of Nursing Sciences, University of Nairobi, University Way, Nairobi, Kenya

DOI: $10.36348 /$ sjnhc.2020.v03i06.003

| Received: 05.06.2020 | Accepted: 13.06.2020 | Published: 16.06.2020

*Corresponding author: Anne Wawire Kabimba

\section{Abstract}

Background: The social society in Africa and most continents, perceives any girl who gives birth before marriage as a wrong doer. Such a girl is deemed to have negated the traditions. Poor and non-communication between adolescents and their parents or guardians has been implicated in most instances. The scenario is the same in the County of study according to information from the respondents and as alluded to by the County Director of health services. The parents and guardians in the County have been called upon to exercise responsibility over their daughters, encourage them to resume school instead of forcefully marrying them off or rejecting them on the bases of pregnancy. The purpose of this study was to give evidence-based health education in relation to family and social support systems in reducing neonatal morbidity and mortality among neonates born to adolescent mothers in the County Referral hospital. Objective: The objective of this study was to describe the role of family and social support in reducing neonatal morbidity and mortality among neonates born to adolescent mothers. Methods: Setting; the study was carried out at a County referral hospital using a randomized control trial design. The participants were expectant adolescent mothers aged 19 years and below attending the antenatal clinic at the hospital. A sample size of 528 respondents (264 cases and 264 controls) was calculated using Pagano formula and realized using simple randomization. Inclusion criteria: adolescent mother $\leq 19$ years, gestation period of 26-34 weeks and resident of the study County for sufficient time follow up. Students, nonKenyans and mothers with existing co-morbidities were excluded for fear of loss to follow up. Results: Negligence and lack of support, $81 \%$ (pv 0.000), lack of community emphasis on neonatal care 77\% (pv0.001), lack of value for neonatal health, 78\% (pv 0.000), lack of knowledge 60\% (pv0.003) and poor socioeconomic status, stigma and rejection $89 \%$ (pv0.000) were some of the significant study findings. Conclusion: The poor family and social support played a major role in persistently high neonatal morbidity and mortality in the County.

Keywords: Support System, Neonates, Neonatal Morbidity/Mortality, Adolescent Mothers.

Copyright @ 2020: This is an open-access article distributed under the terms of the Creative Commons Attribution license which permits unrestricted use, distribution, and reproduction in any medium for non-commercial use (NonCommercial, or CC-BY-NC) provided the original author and source are credited.

\section{BACKGROUND INFORMATION}

The social society in Africa and indeed in most continents, perceives any girl who gets pregnant and gives birth before marriage as a wrong doer. Such a girl is deemed to have negated the culture and traditions. She is therefore liable to punishment as stipulated in the specific cultural norms. Poor or non-communication between the adolescents and their parents or guardians has been implicated in most instances. The scenario is the same in this study according to information from the respondents and as alluded to by the County Director of health services. Severally, the parents and guardians in the County have been called upon to exercise responsibility over their daughters, encourage them to resume school instead of forcefully marrying them off or rejecting them on the basis of being pregnant. The purpose of this study was to implement the evidence- based education in relation to family and social support systems and activities in the reduction of neonatal morbidity and mortality among adolescent mothers.

\section{Specific Objectives}

The study focused on three specific objectives: i). To identify the types and sources of family and social support ii). To describe the role of family and social support systems, iii). To implement and evaluate the impact of evidence-based health education.

\section{Summary of Existing Literature}

In a study on family context, poor communication within families of adolescent mothers was reported as a setback to expected support [1]. In another study carried out in Quang Ninh province, Vietnam it was noted that neonatal morbidity and mortality were never valued by the community [2]. In 
yet another study in Mersin, Turkey it was concluded that the adolescent women had a low perception of social support with the families being the most supportive at a mean score of $23.32 \pm 3.23$ and therefore were never given priority [3]. A study in the United States of America attributed teenage pregnancies and births to single parenthood who lacked proper social learning and integration from immediate families [4]. The adolescent mothers experienced physical, psychological, mental, social challenges and poor social support, increased chances of neonatal morbidity and mortality [5]. Lack of respect, coerced sex, sexual violations, child marriage, sexual abuse and neonatal deaths were all treated with silence in the communities and as such came with consequences to the adolescent mothers [6]. The study on adolescent pregnancy and challenges in Kenya pointed out that most adolescent mothers faced many challenges and lack of social and emotional support, social stigma, poor healthcare access, low economic states and as such were prone to stresses of new life adjustments [7].

In the study County, families and communities were reported as not valuing neonatal health and therefore when illness or death occurred, no efforts were made to respond promptly or report or even register with the registrar of deaths [8]. Devaluing the neonates made it difficult for the referral hospital and the County to keep track and document correct data as many neonatal deaths were never reported. The significance of this study identified the value of individual, family and community awareness regarding neonatal health care practices and the support systems. The study findings informed the health policy makers of the need to adjust to recommendations on neonatal care practices in order to reduce neonatal morbidity and mortality in the County. The results of this study, if adopted have the potential to change the perception of the family and community thus improve the records of the vital statistics to form a data base of records specific to neonates. The study has contributed wealthy knowledge on family, social and adolescent mother interactions.

\section{METHODS}

The study employed a randomized control trial design with allocation ratio of $1: 1$. The target population comprised expectant adolescent mothers aged 19 years and below attending antenatal clinic at the County Referral Hospital in Kenya. The sample size was 452 respondents (226 cases and 226 controls) [9] sampled using simple randomization technique. The data was collected using a semi-structured researcheradministered questionnaire and analyzed using EpiData and STATA version 8.0. Trend analysis was done using logistic regression, frequencies and percentages, precision was set at $95 \%$ CI or 0.05 cut off $(\leq 0.05$ significant, >0.05-insignificant). After data cleaning, the study realized a $14.4 \%$ loss to follow up due wrong contacts and change of residence when the adolescent mothers relocated to their other relatives' homes

\section{Randomization Procedure}

The researcher conducted the whole procedure; generated two small envelopes containing a small unlabeled pink card in one and a white one in another were displayed for the mothers to pick one. Two mothers were given the chance to pick one envelope each at a time. The mothers were not shown the colour of the card they picked so as to conceal the group allocation in order to reduce selection bias and staff influence. Pink cards represented 'Intervention' and white cards represented 'Control'. A sticker of the corresponding color was stuck on the respective mother's antenatal record book. Every expectant adolescent mother meeting the inclusion criteria was selected until the sample size of 264 respondents was attained for each group (a total of 528) and allocated the group accordingly. The mothers and the midwives were both blinded. The two groups had such similar characteristics as; age range, gestation periods, setting (hospital) and health care providers, given same information but different approaches. The respondents were recruited over 36 days during antenatal and followed up through the antenatal period, labor, child birth and neonatal care up to 28 days post birth.

\section{Eligibility Criteria}

Expectant adolescent mothers aged $\leq 19$ years as this group of mothers had not been sufficiently researched on, gestation age, 26-34 weeks allowed the researcher sufficient time for educating the adolescent mothers and following them up to four weeks post childbirth. Being residents of the study County maintained consistence and uniformity of information gathered.

\section{Exclusion Criteria}

Students, none-Kenyans and mothers with comorbidities requiring long term treatment were excluded for fear of 'loss to follow up'. The choice of independent variables was informed by existing literature, reports and records on high rates of neonatal mortality. While the cases were subjected to the evidence-based health education intervention, the controls went through the daily usual routine services of the antenatal clinic, childbirth, postnatal and neonatal care. The main study outcome was 'Reduced neonatal morbidity and mortality'. The interventions for the intervention group (cases) were conducted between March and July 2018. It was assumed that the information gathered was representative of all the adolescent mothers' views and therefore sufficient enough to allow generalization within the County and beyond.

\section{Intervention}

The respondents in the intervention group (cases) were grouped in 20 s based on the gestation 
range; 26-28, 29-31and 32-34 and the corresponding clinic return dates. Each group had three sessions. Each group was taken through sessions on peer, family and social support needs. The objective of this session was to explain the role of support systems during pregnancy, labour, childbirth, postnatal, breast feeding and neonatal care in reducing neonatal morbidity and mortality. The variables addressed included; acceptance of the pregnancy, cultural practices regarding adolescent pregnancy and childbirth, relationships, comfort, overcoming stigma and rejection, physical/emotional and psychological support, counseling, birth preparedness and complication readiness, birth companions and caretakers, place and mode of birth, breastfeeding, finances and coping strategies. The research adopted the interactive teaching method where the researcher gave overviews of the topics, followed by question and answer sessions and sharing experiences. This served to evaluate the understanding of the topic by respondents. The teaching was based on expected/perceived support, sources of support, comfort measures and the importance. The sessions took place in a set aside room for the purpose.

\section{RESULTS}

Baseline data was extracted from 100 maternal antenatal record books randomly picked from the mothers attending the antenatal clinic and 300 most current inpatient files as follows; $50 \%$ of the record books revealed unstable areas of residence as mothers kept changing their physical contacts, $40 \%$ revealed lack of family support as most respondents never had companions to hospital or had entered their grandmothers as their next of kin. 20\% revealed lack of financial support as a major setback. Only in $10 \%$ of the books, the mothers were taught basics likes signs of labour, danger signs but were never given any information on support systems. This was only at a time when nursing students came on board for clinical experience. The files showed that most $(90 \%)$ of the adolescent mothers and their neonates were waived off their bills for lack of financial support. In some files, the neonates had been abandoned probably due to stigma, rejection or lack of where to go with the baby. In $7.6 \%$ of the files, the deceased neonates were never regarded as a problem since some families requested the hospital to dispose them off or simply left the bodies.

In table 1, the respondents' demographic characteristics were summarized. The study identified the following types of support systems from the respondents: i). Parental relationship: as persons who could provide love and affection, ii). Psychological support: acceptance, caring, trust, and counselling, iii). Information support; about pregnancy and management, access to health services, danger signs, childbirth preparedness and complication readiness, breast feeding and newborn care. iv). Peer, community, and social support: where sense of belonging and social identity would be found, find advice, encouragement, social interaction, dialogue, and social learning. v). Physical support: financial assistance, food security, shelter, clothing, and resumption of school. The study identified sources of support as family members, peers, relatives, schools, health workers, charitable organizations, local leaders, and the County Government.

After the intervention, the study results showed that, of the 226 respondents in the intervention group, $34 \%$ went to their own houses since some had families to look after while others considered the comfort, $28.3 \%$ opted to their mothers' houses considering the care, $23.5 \%$ went to other places (mothers in- law, aunties, uncles, friends) and 14.2\% went to their grandmothers houses since there they would be accepted and valued. On the hand, of the 226 respondents in the control group, $38.5 \%$ went to their grandmothers' houses, $34.5 \%$ went to own houses, $6.2 \%$ went to their mothers' houses, $20.8 \%$ opted for other places as did the cases. These findings indicated the state of confusion and desperation the adolescent mothers found themselves in See Figure-1.

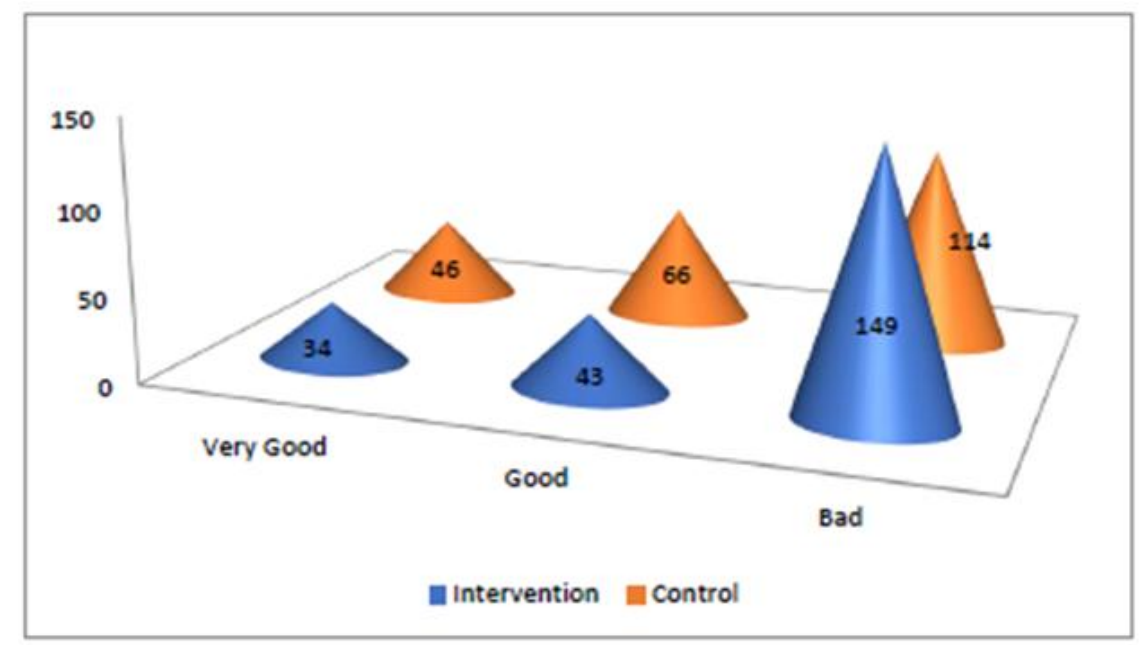

Fig-1: Peer Support System 
Regarding the care of the neonates, the study results from the intervention group showed that $24.3 \%$ of the respondents entrusted the care of their neonates to their mothers due to the confidence. Most, $48.7 \%$ left the neonates with the grandmothers while $22.6 \%$ of the respondents took their neonates along. In the control group, $17.7 \%$ of the respondents left the neonates with their mothers, $53.5 \%$ left the neonates with their grandmothers while $21.2 \%$ of the respondents took their neonates along. The trend is preference to the grandmothers.

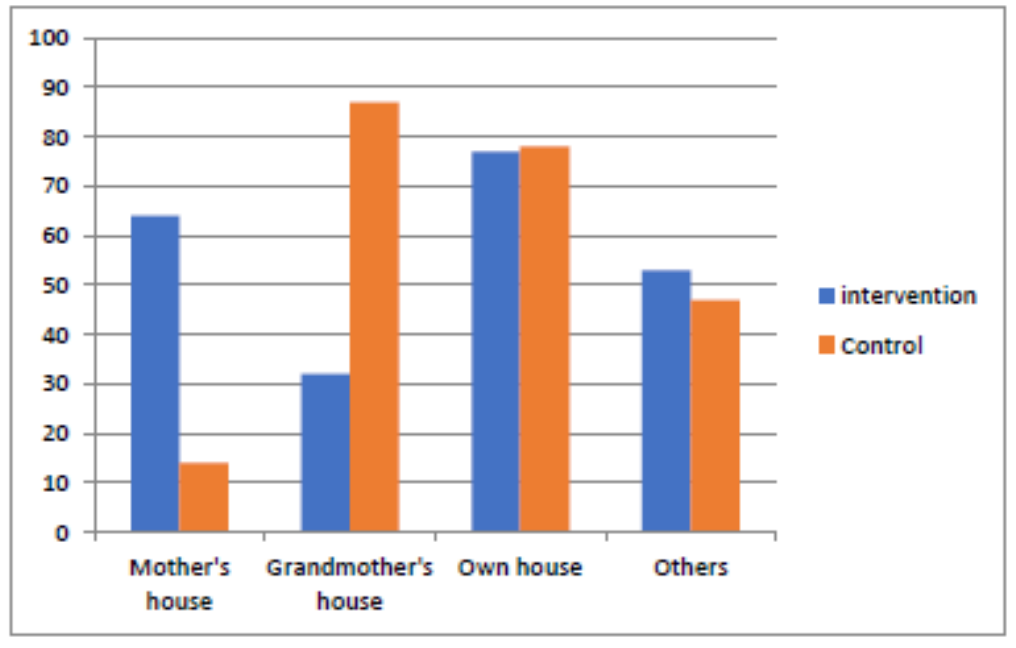

Fig-2: Preference to the grandmothers

Respondents' sustainability was as follows: in the intervention group, $35 \%$ were supported by parents, $19 \%$ did small businesses while $4.9 \%$ depended on salaries/wages and $8.4 \%$ were helped by their partners. In the control group, $34.1 \%$ were supported by parents, $12.8 \%$ depended on businesses while $4.4 \%$ depended on their salaries, Guardians supported $14.6 \%$, friends supported $17.7 \%$, grandmothers, $6.7 \%$. The Financial support was skewed towards parents though minimal.

In regarding the family and social support system: the respondents in the intervention group showed the following: $35.8 \%$ received minimal support, $33.2 \%$ did not receive any support as they were rejected by their families and $30.1 \%$ received adequate support. In the control group, $43.8 \%$ received minimal support, $24.8 \%$ did not receive any support, $27.9 \%$ received adequate support. This indicated that most adolescent mothers were not sufficiently supported.

Regarding peer support in the intervention group, the study results were as follows: 149/226 respondents reported "bad support", 43/226 reported "good support" and 34/226 reported "very good support". In the control group, the study results were as follows: 114/226 reported "bad support", 66/226 reported "good support" and 46/226 reported "very good support" as shown in figure 2. Overly, support from peers was reported as being bad See Figure-2

Respondents in the intervention group reported the following regarding the role of the community in neonatal care: $69.0 \%$ indicated that the community did not value the health of the neonates, $83.6 \%$ reported lack of support to the nursing adolescent mothers, $87.2 \%$ revealed stigmatization and rejection, $56.6 \%$ indicated poor recognition of the ill or diseased neonates, $89 \%$ reported social disconnect and $77 \%$ reported lack of emphasis on neonatal care. In the control group, $70.8 \%$ reported that the community did not value the health of the neonates, $68.6 \%$ reported lack of support to the nursing adolescent mothers, $61.9 \%$ revealed stigmatization and rejection, $55.8 \%$ indicated poor recognition of the ill or diseased neonates, $78.3 \%$ reported lack of emphasis on neonatal care and $90.3 \%$ reported social disconnect. This picture reflects the poor role the community played in the care of the neonates.

\section{Logistic Regression}

At the multivariate level after intervention, social support was less likely to come from the parents as compared to grandmothers, $(\operatorname{Exp}=0.226$, 95\%CI [0.116-0.848], p=0.000). Financial support was less likely to come from spouses as compared to parents $(\mathrm{Exp}=0.262,95 \% \mathrm{CI}[0.067-0.829], \mathrm{p}=0.020)$. Help was more likely to come from health workers when the neonate fell ill or died as compared to family and community $\left(\mathrm{Exp}=0.015,95 \%\right.$ CI [0.015-0.902], $\mathrm{p}_{\mathrm{V}}$ $=0.000)$.

\section{DISCUSSION}

This study identified five types of support systems that were of importance to the adolescent mothers and four areas from which each support was expected to come. These findings were also reported in other studies $[3,10]$. This study further reported that the 
adolescent mothers received minimal support and as such still faced several challenges. Contrary to this finding were the findings in a study in Mersin [4] that reported the teenage mothers having received sufficient support from their parents and relatives. In this study, the peer support was no better with most of the respondents describing it as 'very bad'. The respondents further said that their peers were the ones who usually introduced them to men and into sexual behavior and later abandoned them once they became pregnant. This finding compares with the findings in a study that emphasized the importance of a good family -social support system in order to foster a better life for the adolescent mothers $[11,10]$. Contrary to this finding, a different scenario where the government, teachers, peers, friends and parents worked together to support the adolescent mothers in all aspects including taking care of the neonate so that she could resume studies was reported [4].

This study further reported that, most respondents preferred staying in their own houses after childbirth if possible. Otherwise they would stay with their grandmothers for protection. However, the rest of the mothers after childbirth, with any other willing persons. The reasons to this type of movement were blamed on the poor social support system. To support this finding, it was argued that a good social supportive system played a vital role in molding the adolescent mothers in self and eventual neonatal care [12].

Concerning care of the neonates, this study found out that most adolescent mothers left their neonates under the care of grandmothers. The reasons were probably due to lack of knowledge and skills, in ability to cope with the demands of the neonate and lack of psychological support but they were also accommodative and sympathetic. This finding was also reported in a study where the adolescent mothers maintained that if it were not for their grandmothers and aunties, life would have been impossible to manage when caring for their newborns [12]. This finding was further supported by findings in another study that examined the grandmothers' capacities to care for the neonates and noted that grandmothers impacted positively in the adolescents' neonatal care period [13].

Most respondents in this study reported that health care providers played a vital role in supporting them during neonatal illness or bereavement as compared to the family members and the community. In fact, the culture prohibited people from mourning a neonate as it was believed that mourning prevented another baby being born. Where one twin died, it was kept extremely silent to protect the remaining twin. The adolescent mother was closely monitored in case she shed tears over the living twin. Because of this negative attitude, even the adolescent mothers also attempted to or abandoned the dead neonates in the hospital beds.
This study reported that male partners denied the pregnancies, the babies, and any liability. This finding was supported by a study carried out in Kenya which revealed that male partners of the adolescent mothers negligibly supported them [7].

The community in this study was perceived as not supportive and therefore subjected the adolescent mothers to stigma and rejection. These findings concurred with the findings in several studies which reported that social stigma, lack of affection and emotional support, stresses and new life adjustments were issues that adversely affected the adolescent mothers of which they needed sufficient support $[1,7$, $14,15]$.

Study Limitations: Inaccurate record keeping, wrong contacts, limited finances.

\section{CONCLUSION}

The poor family and social support to the adolescent mothers played a major role in persistently high neonatal morbidity and mortality in the County Referral Hospital. The study findings if adopted would inform the County Health Policy makers of the need to adjust to interventions for the reduction of neonatal morbidity and mortality. Similarly, community awareness would change the traditional perception of care of the neonate. The study helped to improve the record keeping of the vital statistics thus forming a database for records specific to neonates and as well contributed wealthy knowledge on family and social roles and interactions.

\section{Availability of Data and Materials}

The researcher obtained permissions from the County Referral Hospital. More information was extracted from the Kenya Demographic Survey 2014 document.

\section{Abbreviations \\ CI: Confidence interval}

Declaration: Ethics approval, consent to participate and approval letter as attached.

Competing interests: The author has no competing interests. The content in this study is the author's original research work.

\section{Funding: Self}

\section{ACKNOWLEDGEMENT}

I am deeply indebted to the following persons: my able supervisors: Emmah Matheka and Sabina Wakasiaka for their exemplary supervision, mentorship and guidance, patience, and encouragement. Many thanks to reviewers, statisticians and fellow $\mathrm{PhD}$ Students for their inputs and support. I will forever hold 
my family in high esteem for being so patient, supportive and encouraging.

Author's Information: This article is a revised version of a sub-section of my $\mathrm{PhD}$ thesis (Specific Objective 2).

Consent for publication: Individual data was not presented therefore not applicable.

\section{REFERENCES}

1. Sámano, R., Martínez-Rojano, H., Robichaux, D., Rodríguez-Ventura, A. L., Sánchez-Jiménez, B., de la Luz Hoyuela, M., ... \& Segovia, S. (2017). Family context and individual situation of teens before, during and after pregnancy in Mexico City. BMC pregnancy and childbirth, 17(1), 382.

2. Målqvist, M. (2011). Neonatal mortality: an invisible and marginalised trauma. Global health action, 4(1), 5724.

3. Yurdakul, M. (2018). Perceived social support in pregnant adolescents in Mersin area in Turkey. Pakistan journal of medical sciences, 34(1), 115-120.

4. Akella, D., \& Jordan, M. (2011). Impact of social and cultural factors on teenage pregnancy. Journal of Health Disparities Research and Practice; 8(1):41-62.

5. Mangeli, M., Rayyani, M., \& Tirgat, B. (2017). Explorirng challenges of Adolescent Mothers from their Life Experiences in the transition to Motherhood: A Qualitative Study. J Fam and Reprod Health, 11(3):165-73.

6. National Adolescent Sexual and Reproductive Health Policy, Reaffirming Support for Adolescent Sexual and Reproductive Health, 2015, Ministry of Health, Kenya.

7. Kumar, M., Huang, K. Y., Othieno, C., Wamalwa, D., Madeghe, B., Osok, J., ... \& McKay, M. M. (2018). Adolescent pregnancy and challenges in Kenyan context: Perspectives from multiple community stakeholders. Global Social

Welfare, 5(1), 11-27.

8. Kenya Demographic and Health Survey, 2014. Ministry of Health, Nairobi, Kenya.

9. Pagano, M., \& Gauvreau, K. (2000). Principles of Biostatistics, $2^{\text {nd }} e d$. Duxbury Press. ISBN-13:9780534229023.

10. Letourneau, N. L., Stewart, M. J., \& Barnfather, A. K. (2004). Adolescent mothers: Support needs, resources, and support-education interventions. Journal of adolescent health, 35(6), 509-525.

11. Watts, M. C. N. C., Liamputtong, P., \& Mcmichael, C. (2015). Early motherhood: a qualitative study exploring the experiences of African Australian teenage mothers in greater Melbourne, Australia. BMC public health, 15(1), 873.

12. Leech, T. G., Adams, E. A., \& Littlefield, M. (2014). A community conversation on adolescent pregnancy and parenting services: networks of support, gatekeepers to care, and non-compulsory fathering in a black urban community. Journal of Health Disparities Research and Practice, 7(7):46-62.

13. Wilson, D., Gross, D., Hodgkinson, S., \& DeaterDeckard, K. (2017). Association of teen mothers' and grandmothers' parenting capacities with child development: A study protocol. Research in nursing \& health, 40(6), 512-518.

14. Neal, S., Channon, A. A., \& Chintsanya, J. (2018). The impact of young maternal age at birth on neonatal mortality: Evidence from 45 low and middle income countries. PloS one, 13(5): 0195731.

15. Miura, P. O., Tardivo, L. S. D. L. P., \& Barrientos, D. M. S. (2018). Helplessness experienced by adolescent mothers and pregnant adolescents sheltered in institutions. Ciencia \& saude coletiva, 23(5), 1601-1610. 